\section{(C) OPEN ACCESS}

\title{
The MITOS system predicts long-term survival in amyotrophic lateral sclerosis
}

\author{
Irene Tramacere, ${ }^{1}$ Eleonora Dalla Bella, ${ }^{2}$ Adriano Chiò, ${ }^{3}$ Gabriele Mora, ${ }^{4}$ \\ Graziella Filippini, ${ }^{1}$ Giuseppe Lauria, ${ }^{2}$ on behalf of the EPOS Trial Study Group
}

\begin{abstract}
- Additional material is
published online only. To view please visit the journal online (http://dx.doi.org/10.1136/ jnnp-2014-310176).

${ }^{1}$ Neuroepidemiology Unit, IRCCS Foundation, "Carlo Besta" Neurological Institute, Milan, Italy

${ }^{2} 3$ rd Neurology Unit, Motor Neuron Diseases Centre, IRCCS Foundation, "Carlo Besta" Neurological Institute, Milan, Italy

${ }^{3}$ Department of Neurosciences, ALS Centre, "Rita Levi Montalcini", University of Turin and Azienda Ospedaliero Universitaria Città della Salute e della Scienza, Turin, Italy ${ }^{4}$ IRCCS "Salvatore Maugeri" Foundation, Milan, Italy
\end{abstract}

\section{Correspondence to} Dr Giuseppe Lauria, 3rd Neurology Unit, Motor Neuron Diseases Centre, IRCCS Foundation, "Carlo Besta" Neurological Institute, Via Celoria, 11, Milan 20133, Italy; glauria@istituto-besta.it

Received 17 December 2014 Revised 11 March 2015 Accepted 30 March 2015 Published Online First 17 April 2015
CrossMark

\footnotetext{
To cite: Tramacere I, Dalla Bella E, Chiò A, et al. J Neurol Neurosurg Psychiatry 2015;86:11801185.
}

\section{ABSTRACT \\ Objective The choice of adequate proxy for long-term} survival, the ultimate outcome in randomised clinical trials (RCT) assessing disease-modifying treatments for amyotrophic lateral sclerosis (ALS), is a key issue. The intrinsic limitations of the ALS Functional Rating ScaleRevised (ALSFRS-R), including non-linearity, multidimensionality and floor-effect, have emerged and its usefulness argued. The ALS Milano-Torino staging (ALS-MITOS) system was proposed as a novel tool to measure the progression of ALS and overcome these limitations. This study was performed to validate the ALS-MITOS as a 6-month proxy of survival in 200 ALS patients followed up to 18 months.

Methods Analyses were performed on data from the recombinant human erythropoietin RCT that failed to demonstrate differences between groups for both primary and secondary outcomes. The ALS-MITOS system is composed of four key domains included in the ALSFRS-R scale (walking/self-care, swallowing, communicating and breathing), each with a threshold reflecting the loss of function in the specific ALSFRS-R subscores. Sensitivity, specificity and the area under the curve of the receiver operating characteristic curves of the ALS-MITOS system stages and ALSFRS-R decline at 6 months were calculated and compared with the primary outcome (survival, tracheotomy or >23-hour noninvasive ventilation) at 12 and 18 months Predicted probabilities of the ALS-MITO system at 6 months for any event at 12 and 18 months were computed through logistic regression models.

Results Disease progression from baseline to 6 months as defined by the ALS-MITOS system predicted death, tracheotomy or $>23$-hour non-invasive ventilation at 12 months with $82 \%$ sensitivity $(95 \% \mathrm{Cl} 71 \%$ to $93 \%$, $n=37 / 45)$ and $63 \%$ specificity $(95 \% \mathrm{Cl} 55 \%$ to $71 \%$, $\mathrm{n}=92 / 146)$, and at 18 months with $71 \%$ sensitivity $(95 \% \mathrm{Cl} 61 \%$ to $82 \%, n=50 / 70)$ and $68 \%$ specificity (95\% Cl $60 \%$ to $77 \%, n=76 / 111)$. The analysis of ALSMITOS and ALSFRS-R progression at 6-month follow-up showed that the best cut-off to predict survival at 12 and 18 months was 1 for the ALS-MITOS (ie, loss of at least one function) and a decline ranging from 6 to 9 points for the ALSFRS-R.

Conclusions The ALS-MITOS system can reliably predict the course of ALS up to 18 months and can be considered a novel and valid outcome measure in RCTs.

\section{INTRODUCTION}

Amyotrophic lateral sclerosis (ALS) is a fatal neurodegenerative disease leading to the majority of patients dying within a limited number of years. ${ }^{12}$
The disability score ALS Functional Rating Scale-Revised (ALSFRS-R) ${ }^{3}$ has been used to measure the course of the disease and to assess the efficacy of candidate treatments in clinical trials. ${ }^{4-8}$ However, its ability to be a reliable outcome in disease-modifying clinical trials has been argued due to the following limitations: (1) non-linear, thus prone to biases; ${ }^{9}$ (2) multidimensional, thus unfit as single score and unable to satisfy rigorous measurement standards; ${ }^{9}$ (3) floor-effect, thus unable to capture late-stage clinical changes. ${ }^{1} 1011$

Recently, the ALS Milano-Torino staging (ALS-MITOS) system was proposed as a novel tool to measure the progression of ALS. $^{1}$ The ALS-MITOS system, which is based on the assessment of four functional domains assayed by the ALSFRS-R, was found to reliably identify relevant stages of disease in patients according to the number of functions lost, to be consistent with sequential disease progression, to overcome nonlinearity and multidimensionality features of ALSFRS-R, and to correlate well with patients' quality of life and health service costs. ${ }^{1}$ This study aimed to confirm the robustness of the ALS-MITOS system and validate its ability to be a proxy of long-term survival.

\section{METHODS}

Patients and measures

Data were derived from a randomised, doubleblind, placebo-controlled clinical trial (RCT) on the efficacy of recombinant human erythropoietin in ALS that failed to demonstrate differences between active treatment and placebo, in primary and in secondary outcomes. ${ }^{12}$ Briefly, patients aged 18-75 years and diagnosed with probable laboratory-supported, probable or definite ALS according to El Escorial revised criteria were enrolled in 25 Italian ALS centres. Inclusion criteria were onset $\leq 18$ months and slow vital capacity $\geq 70 \%$ of predicted value at the screening visit. The primary outcome was time to death, tracheotomy or $>23 \mathrm{~h}$ non-invasive ventilation (NIV) daily for 14 consecutive days at 12 months. Development and evaluation of the ALS-MITOS system have been previously described. ${ }^{1}$ Briefly, four key domains included in the ALSFRS-R scale (walking/ self-care, swallowing, communicating and breathing) were selected to obtain a tool capable of capturing the lost functions. Each domain has a threshold reflecting the loss of function in the specific ALSFRS-R subscores. Values of 0 (below threshold) or 1 (above threshold) were assigned, 
and the stages were determined as the sum of those values across the four domains. Five stages were defined: stage 0 indicates functional involvement and no loss of function in any domain; stages 1-4 represent the number of domains in which function was lost; and stage five is death (see online supplementary table). ${ }^{1}$

\section{Statistical analysis}

Baseline patients' features were reported as percentages for dichotomous data, means with SD and medians with value range for continuous data. The ALS-MITOS system was reported using descriptive statistics for each participant at baseline, and at 6-month and 12-month follow-up. Sensitivity and specificity with the corresponding $95 \% \mathrm{CI}$, and the area under the curve of the receiver operating characteristic (ROC) curves of the ALS-MITOS system stages as well as ALSFRS-R decline at 6 months were calculated, and compared to the primary

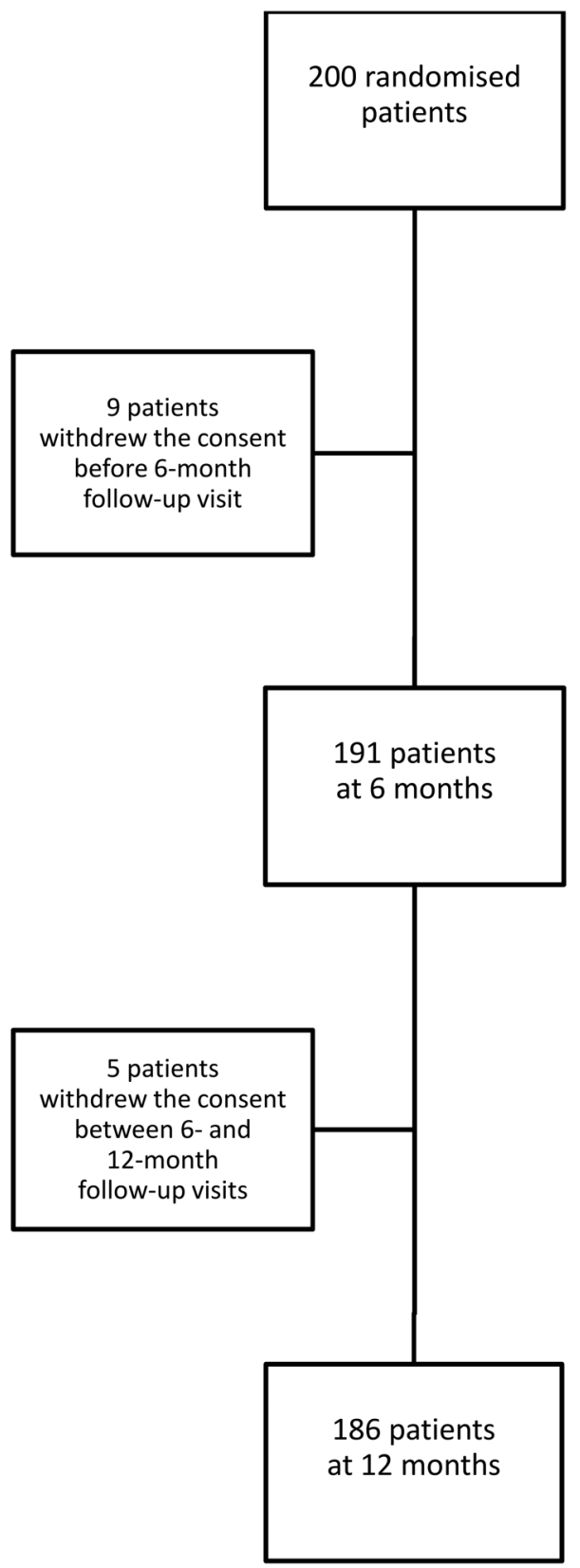

Figure 1 Flow-chart. outcome (survival, tracheotomy or $>23 \mathrm{~h} \mathrm{NIV)} \mathrm{at} 12$ and 18 months, where an event was defined by the occurrence of at least one among the three. Predicted probability with the corresponding 95\% CI of the ALS-MITO system at 6 months for any event at 12 and 18 months was computed through logistic regression models.

\section{RESULTS}

Among the 200 patients randomised, nine withdrew consent before 6-month follow-up and five between 6-month and 12-month follow-up (figure 1). Table 1 reports patients' demographic and clinical features at the baseline visit. In the analysis of progression based on the ALS-MITOS system, 153 of 200 patients $(76.5 \%)$ at baseline were in stage 0 and 44 patients $(22 \%)$ were in stage 1 , while only three patients $(1.5 \%)$ were in stage 2 and none in stage 4 . Among the 44 patients in stage 1 , $38(86.4 \%)$ had lost function in walking/self-care, $3(6.8 \%)$ in breathing, $2(4.6 \%)$ in swallowing and $1(2.3 \%)$ in communicating. Among the 191 patients who performed the 6-month follow-up visit or died, 91 (47.6\%; 49.7\% of 141 spinal and $42 \%$ of 50 bulbar onset patients, $\mathrm{p}=0.35$ ) progressed to advanced stages of disease (figure 2A). Among the 186 patients who performed the 12-month follow-up visit or died, 136 (73.1\%; $74.5 \%$ of 137 spinal and $69.4 \%$ of 49 bulbar onset patients, $\mathrm{p}=0.49$ ) progressed to advanced stages of disease and $50(26.9 \%)$ did not. Overall, 42 patients $(22.6 \%)$ were in stage 0,50 patients $(26.9 \%)$ were in stage 1,34 patients $(18.3 \%)$ were in stage 2,29 patients $(15.6 \%)$ were in stage 3 , nine patients $(4.8 \%)$ were in stage 4 and 22 patients $(11.8 \%)$ were in stage 5 (death). At 12 months, among the 122 subjects with at least one function lost, $109(89.3 \%)$ had loss of autonomy in walking/self-care, 56 (45.9\%) in breathing, 44 (36.1\%) in swallowing and $32(26.2 \%)$ in communicating. Patients at stage 1 at baseline had a higher, although non-statistically significant, probability of transition to advanced stages $(75 \%)$ or of dying $(20 \%)$ than those at stage $0(70.6 \%$ and $9.8 \%$, respectively, $\mathrm{p}=0.07$ ) (figure $2 \mathrm{~B}$ ). No transition to a lower stage of disease was observed between baseline and 12 months (two patients moved from stage 1 at baseline to stage 0 at 6 months and returned to stage 1 at 12 months, due to fluctuations in walking/ self-care domain).

Table 1 Baseline characteristics

\begin{tabular}{ll}
\hline Characteristic & $\mathrm{N}=\mathbf{2 0 0}$ \\
\hline $\begin{array}{l}\text { Demographic characteristics } \\
\text { Female, } \mathrm{n}(\%)\end{array}$ & $95(47.5 \%)$ \\
Age, years & \\
$\quad$ Mean \pm SD & $59 \pm 10$ \\
$\quad$ Median (range) & $61(25-75)$ \\
Clinical characteristics & \\
Time since ALS onset, years & \\
$\quad$ Mean \pm SD & $1.0 \pm 0.4$ \\
$\quad$ Median (range) & $1.1(0.2-1.7)$ \\
ALS onset type, $n$ (\%) & \\
$\quad$ Spinal & $148(74.0 \%)$ \\
$\quad$ Bulbar & $52(26.0 \%)$ \\
ALSFRS-R score at entry & \\
$\quad$ Mean \pm SD & $38.3 \pm 5.8$ \\
$\quad$ Median (range) & $39(20-48)$ \\
\hline ALS, amyotrophic lateral sclerosis; ALSFRS-R, Amyotrophic Lateral Sclerosis Functional \\
Rating Scale-Revised.
\end{tabular}




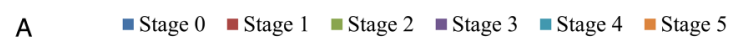

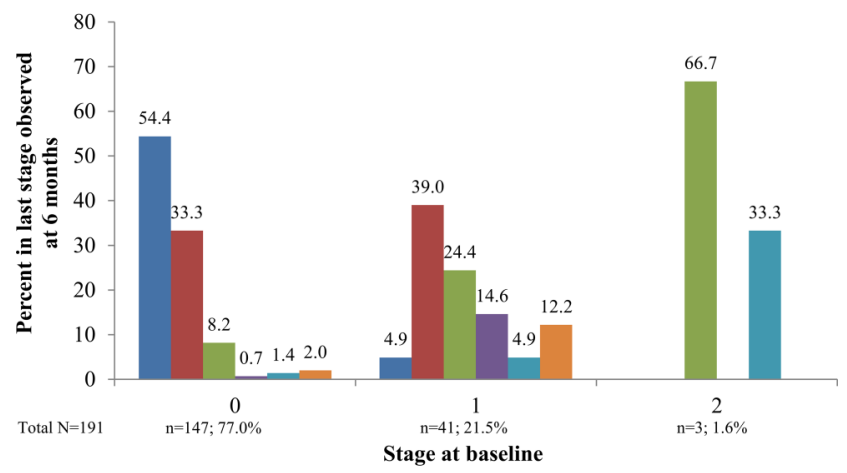

B $\square$ Stage $0 \quad \square$ Stage $1 \quad \square$ Stage $2 \backsim$ Stage $3 \backsim$ Stage $4 \backsim$ Stage 5

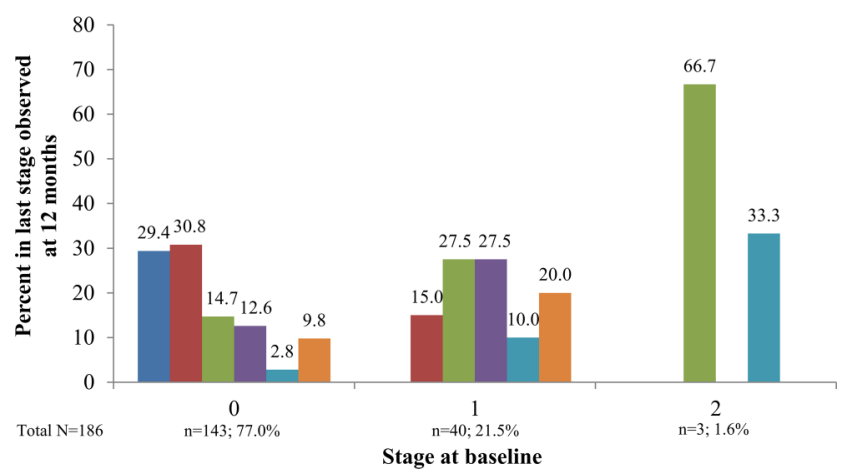

Figure 2 ALS stage at 6 months (A) and at 12 months (B) by baseline stage. ALS, amyotrophic lateral sclerosis.

The analysis for any stage of disease progression from baseline to 6-month follow-up showed sensitivity of $82 \%$ (95\% CI $71 \%$ to $93 \%, n=37 / 45)$, specificity of $63 \%(95 \%$ CI $55 \%$ to $71 \%$, $\mathrm{n}=92 / 146)$, a positive predictive value of $41 \%$ and a negative predictive value of $92 \%$ of the ALS-MITOS system for the primary outcome (death, tracheotomy or $>23 \mathrm{~h} \mathrm{NIV)} \mathrm{at}$ 12 months. In other words, based on the disease worsening at 6 months from the first observation at baseline, irrespective of the number of functions lost at 6 months, the ALS-MITOS system was able to correctly identify one patient who would have an event included in the primary outcome at 12 months with a probability of $82 \%$. At the same time, the system was able to correctly identify with a probability of $63 \%$ one patient who would not have an event included in the primary outcome. When we analysed the number of functions lost at 6 months compared with baseline, the ALS-MITOS system was found to have a probability to predict the primary outcome at 12 months with sensitivity of $62 \%$ and specificity of $67 \%$ in patients who advanced by 1 stage (eg, from stage 0 to 1 , or from 1 to 2 , etc), and of $62 \%$ and $94 \%$, respectively, in those who advanced by two stages (eg, from stage 0 to 2 , or from 2 to 4 , etc). The corresponding positive predictive values were $22 \%$ for patients who advanced by one stage and $68 \%$ for those who advanced by two stages, showing a higher probability to have an event at 12 months for patients who advanced by two stages compared to a progression of one stage.

The corresponding analysis at 18 months for any stage of progression from baseline to 6 months showed sensitivity of $71 \%$ (95\% CI $61 \%$ to $82 \%, \mathrm{n}=50 / 70)$, specificity of $68 \%(95 \% \mathrm{CI}$ $60 \%$ to $77 \%, n=76 / 111$ ), a positive predictive value of $59 \%$ and a negative predictive value of $79 \%$ of the ALS-MITOS system for the primary outcome (death, tracheotomy or $>23 \mathrm{~h}$
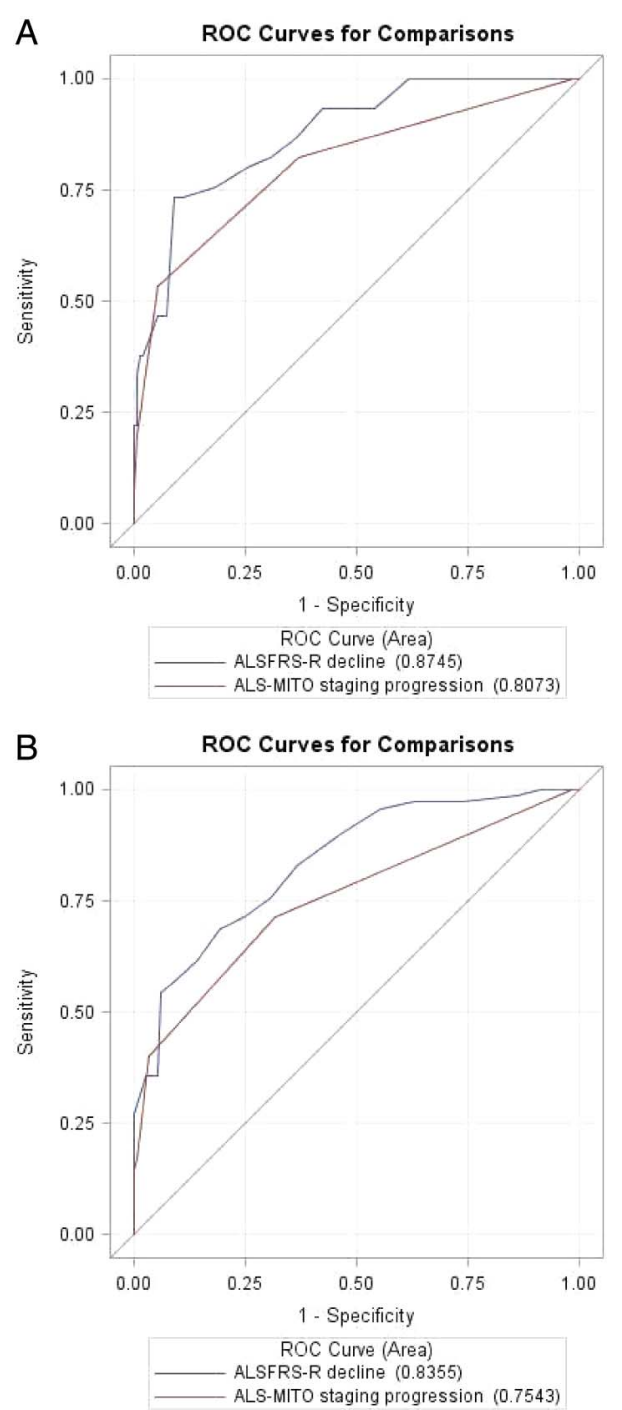

Figure 3 ROC curves for (A) ALSFRS-R decline and ALS-MITO staging progression from baseline to 6 months versus primary outcome at 12 months, and (B) ALSFRS-R decline and ALS-MITO staging progression from baseline to 6 months versus primary outcome at 18 months. ALS, amyotrophic lateral sclerosis; ALSFRS-R, Amyotrophic Lateral Sclerosis Functional Rating Scale-Revised; ALS-MITO, ALS Milano-Torino ; ROC, receiver operating characteristic.

NIV). When we analysed the number of functions lost at 6 months compared with baseline, the ALS-MITOS system was found to have a probability to predict the primary outcome at 18 months with sensitivity of $52 \%$ and specificity of $71 \%$ in patients who advanced by one stage (eg, from stage 0 to 1 , or from 1 to 2 , etc), and of $44 \%$ and $96 \%$, respectively, in those who advanced by two stages (eg, from stage 0 to 2 , or from 2 to 4 , etc)

The comparison between ALS-MITOS system progression and ALSFRS-R decline over the first 6 months from baseline showed that the area under the ROC curves of the ALSFRS-R decline was slightly higher than that of the ALS-MITOS system progression at both 12 months (0.87 and 0.81 , respectively) and 18 months (0.84 and 0.75, respectively) (figure 3). The corresponding values adjusted by the baseline ALSFRS-R score did not significantly change $(0.88$ and 0.83 for the ALSFRS-R and ALS-MITOS system, respectively, at 12 months; 0.85 and 0.79 for the ALSFRS-R and ALS-MITOS system, respectively, at 18 months). Based on these curves, the best 
Table 2 Sensitivity and specificity, with the corresponding $95 \% \mathrm{Cl}$, of the ALSFRS-R decline and the ALS-MITOS worsening from baseline to 6 months compared to the primary composite outcome (ie, survival, tracheotomy or $>23 \mathrm{~h}$ non-invasive ventilation) at 12 and 18 months

\begin{tabular}{|c|c|c|c|c|}
\hline \multirow[b]{2}{*}{ Scale } & \multicolumn{2}{|c|}{ Compared to primary outcome at 12 months } & \multicolumn{2}{|c|}{$\begin{array}{l}\text { Compared to primary outcome at } \\
18 \text { months }\end{array}$} \\
\hline & $\begin{array}{l}\text { Sensitivity } \\
(95 \% \mathrm{Cl})\end{array}$ & $\begin{array}{l}\text { Specificity } \\
(95 \% \mathrm{Cl})\end{array}$ & $\begin{array}{l}\text { Sensitivity } \\
(95 \% \mathrm{Cl})\end{array}$ & $\begin{array}{l}\text { Specificity } \\
(95 \% \mathrm{Cl})\end{array}$ \\
\hline \multicolumn{5}{|c|}{ 6-month ALSFRS-R decline } \\
\hline 6 points & $\begin{array}{l}93 \% \\
(86 \% \text { to } 100 \%)\end{array}$ & $\begin{array}{l}58 \% \\
(50 \% \text { to } 66 \%)\end{array}$ & $\begin{array}{l}83 \% \\
\text { (74\% to } 92 \%)\end{array}$ & $\begin{array}{l}63 \% \\
\text { (54\% to } 72 \%)\end{array}$ \\
\hline 7 points & $\begin{array}{l}87 \% \\
\text { (77\% to } 97 \%)\end{array}$ & $\begin{array}{l}64 \% \\
(56 \% \text { to } 72 \%)\end{array}$ & $\begin{array}{l}76 \% \\
(66 \% \text { to } 86 \%)\end{array}$ & $\begin{array}{l}69 \% \\
\text { (61\% to } 78 \%)\end{array}$ \\
\hline 8 points & $\begin{array}{l}82 \% \\
(71 \% \text { to } 93 \%)\end{array}$ & $\begin{array}{l}69 \% \\
(62 \% \text { to } 77 \%)\end{array}$ & $\begin{array}{l}71 \% \\
(61 \% \text { to } 82 \%)\end{array}$ & $\begin{array}{l}75 \% \\
(67 \% \text { to } 83 \%)\end{array}$ \\
\hline 9 points & $\begin{array}{l}80 \% \\
(68 \% \text { to } 92 \%)\end{array}$ & $\begin{array}{l}75 \% \\
(68 \% \text { to } 82 \%)\end{array}$ & $\begin{array}{l}69 \% \\
(58 \% \text { to } 79 \%)\end{array}$ & $\begin{array}{l}80 \% \\
\text { (73\% to } 88 \%)\end{array}$ \\
\hline \multicolumn{5}{|c|}{ 6-month ALS-MITOS system worsening } \\
\hline 1 function & $\begin{array}{l}82 \% \\
(71 \% \text { to } 93 \%)\end{array}$ & $\begin{array}{l}63 \% \\
(55 \% \text { to } 71 \%)\end{array}$ & $\begin{array}{l}71 \% \\
(61 \% \text { to } 82 \%)\end{array}$ & $\begin{array}{l}68 \% \\
\text { (60\% to } 77 \%)\end{array}$ \\
\hline
\end{tabular}

cut-off of the ALS-MITOS system to predict at 6 months the primary outcome at 12 and 18 months was one (ie, loss of at least one function from baseline to 6 months), whereas that of the ALSRFS-R ranged between six to nine points of decline (table 2).

As expected, we observed that the higher the stage of disease based on the ALS-MITOS system at 6 months, the higher the probability to reach the primary outcome within 12 and 18 months (figure 4). Irrespective of the stage of the disease at
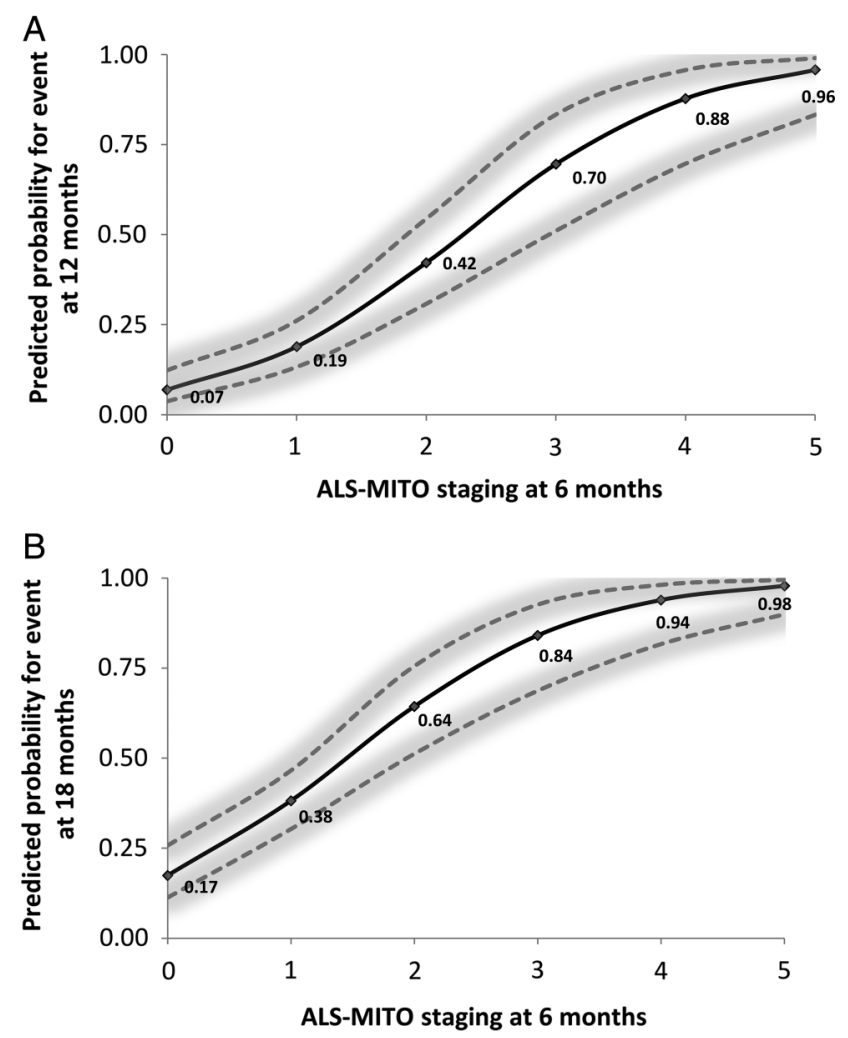

Figure 4 Predicted probability with the corresponding $95 \% \mathrm{Cl}$ of ALS-MITO staging at 6 months for event at 12 months (A) and at 18 months (B). ALS, amyotrophic lateral sclerosis; ALS-MITO, ALS Milano-Torino.
6 months, the predicted probability to have an event included in the primary outcome at 18 months was always higher than at 12 months. For example, patients who had lost two functions at 6 months had a predicted probability of about $40 \%$ to have an event of the primary outcome within 12 months and of about $65 \%$ within 18 months.

\section{DISCUSSION}

The present study confirmed the validity and robustness of the ALS-MITOS system to be consistent with sequential disease progression and revealed its ability to be a proxy of long-term outcome. Moreover, it has demonstrated reliability and ease of use across a large number of ALS centres. In particular, we found that, according to the ALS-MITOS system, in our population of 200 patients with ALS with an onset $\leq 18$ months, almost $50 \%$ at 6 months and more than $70 \%$ at 12 -month follow-up progressed to advanced stages of disease. According to previous findings, ${ }^{1}$ the probability of transition from a given stage was highest for the next higher stage, with the majority of patients reporting a loss of function in walking/self-care (89\%), almost 50\% in breathing and more than 30\% in swallowing, at 12 months.

The progression of disease from baseline to 6 months as defined by the ALS-MITOS system appeared to be a good proxy of death, tracheotomy or $>23 \mathrm{~h} \mathrm{NIV}$ at 12-month and 18 -month follow-up in terms of sensitivity and predicted probability. Furthermore, the ALS-MITOS system correlated well with the 6-month ALSFRS-R decline, showing similar accuracy. Accordingly, the analysis of the ROC curves demonstrated that, compared to the best cut-off values of the ALSFRS-R decline over 6 months (ie, values within the range from six to nine, where six would be the best cut-off to increase sensitivity), the best cut-off values of the ALS-MITOS system showed satisfactory sensitivity and specificity values. Moreover, it should be considered that there is no validated cut-off for the ALSFRS-R and, mostly, that the clinical meaningfulness of a difference in one or two points can be influenced by the baseline score of the study population and, overall, may be hardly considered clinically meaningful.

During the past 7 years, 18 RCTs $^{4-6}{ }^{12-26}$ on 14 different drugs were performed and failed to show positive results. Although progressively better organised, all these RCTs showed 
similar issues, which could have influenced the negative findings, including the choice of the outcomes. ${ }^{27} 28$ The ultimate end point in ALS is the time to death; however, it is not a feasible outcome unless very long trials are designed. Indeed, more than 95\% of patients enrolled in RCTs were alive at 6 months and 75-90\% at 12 months. ${ }^{4-6} 12-1417182123$ As emphasised in the TCH346 trial, ${ }^{24}$ different clinical practice can influence survival, beyond the need of more than 1000 patients followed up for more than 3 years, to have an adequate power to use it as primary outcome. To overcome this issue, that and many other trials $^{1821222425}$ chose the mean ALSFRS-R decline as primary outcome. However, ALSFRS-R also showed major limitations, beyond the already mentioned non-linearity, multidimensionality and inability to detect late-stage clinical changes. ${ }^{1}{ }^{9-11}$ Indeed, a mean decline of one point/month up to 12 months from randomisation was reported in all the longitudinal studies, ${ }^{4-6} 12-26$ and a plateau between 12 and 18 months in the only two studies including both those follow-up periods, ${ }^{6} 141617$ suggesting that the mean ALSFRS-R decline cannot serve as a reliable outcome either at 6 months, since only differences of one to three points between treatment groups can be observed, or at 18 months, being unable to capture slower changes in later stages. Consistently, the authors of the glatiramer acetate trial conducted on 366 patients with ALS, ${ }^{21}$ speculated that the choice of the ALSFRS-R decline as primary outcome might be one of the reasons underlying the negative results of the study, likely being either insensitive or inappropriate.

A further important problem of the ALSFRS-R is how to consider deaths. Accordingly, in the CoQ10 trial, ${ }^{22}$ the significantly lower decline of the mean ALSFRS-R observed in the CoQ10-treated arm was almost entirely driven by outlying values from five deceased placebo patients, while the median decline that is insensitive to outliers, was lower in the placebo arm. Consistently, in the dexpramipexole trial, ${ }^{5}$ the authors used as primary outcome a combined assessment of survival and change from baseline in the ALSFRS-R score, emphasising that the ALSFRS-R decline alone could not be proposed as primary outcome due to the well known non-linearity over time, and because it should be assumed that discontinuations, including deaths, were random and non-informative.

Combined outcome measures including survival, tracheotomy, NIV and/or selected domains of the ALSFRS-R scale showed better performances compared to survival or mean ALSFRS-R decline alone in several studies. ${ }^{4} 512171920$ For example, in one of the lithium trials, ${ }^{19}$ the composite outcome of severe loss of autonomy, defined as the time from inclusion to death/tracheotomy or at least two of three selected ALSFRS-R scores (ie, $\leq 1$ for swallowing, $\leq 1$ for walking, or $\leq 2$ for respiratory insufficiency), was able to capture a percentage of events around $15 \%$ at 6 months and above $30 \%$ at 12 months. Similarly, in the acetyl-L-carnitine trial, ${ }^{4}$ the composite primary outcome of loss of self-sufficiency, defined as inability to swallow, cut food/ handle utensils and walk, was observed in more than $80 \%$ of patients at 12 months.

The ALS-MITOS system ${ }^{1}$ was developed to be a reliable composite outcome measure. The present study, performed to validate its use in RCTs, demonstrated its good correlation with death, tracheotomy or $>23 \mathrm{~h} \mathrm{NIV}$, as well as with the mean ALSFRS-R decline over 6 months. Moreover, the ALS-MITOS system can be a more clinically meaningful outcome in shortterm and medium-term trials compared with ALSFRS-R. Indeed, it is based on the unidirectional progression of function impairment in four key domains (walking/self-care, swallowing, communicating and breathing), the achievement of which means their loss without any possibility to recover.

What an ALS trial is expected to demonstrate in a time frame useful to patients is that the candidate treatment can slow (or hopefully halt) the progression of the disease. While it is arguable to consider a drug that only prolongs survival by a few months as efficacious, a difference of two-point decline over 6 months at the ALSFRS-R can hardly be considered clinically meaningful. Moreover, the ALSFRS-R is not unequivocally unidirectional, typically showing fluctuations over time that can be due to treatments ameliorating certain symptoms (eg, drooling). Conversely, a difference in terms of function lost or retained can more reliably reflect the real effect of a treatment in ALS. Our findings demonstrated that the ALS-MITOS system can reliably correlate loss of functions at 6 months to survival at 12 and 18 months. Meeting the requirements of modern approaches to ALS trials, ${ }^{28}$ it can be considered a novel and valid outcome measure.

Acknowledgements The authors thank all the patients and their families, and the Fondazione Italiana di Ricerca per la SLA - Sclerosi Laterale Amiotrofica (AriSLA). The authors thank Domenico Arenella and Laura Ferradini for administrative and coordination assistance, and the entire study group for the participation.

Collaborators EPOS Trial Study Group: Giovanni Antonini (NESMOS Dept, Neuromuscular Disease Unit, Sant'Andrea Hospital and University of Rome 'Sapienza', Rome), Giuseppe Borghero (Neurologic Unit, Monserrato University Hospital, Cagliari), Margherita Capasso (Neurologic Clinic, SS Annunziata Hospital, Chieti), Claudia Caponnetto (Neurosciences, Rehabilitation Ophthalmology, Genetics, Mother and Child Disease Dept, IRCCS University Hospital San Martino IST, Genova), Christian Lunetta, Massimo Corbo* (NEMO Clinical Centre, Milan [*actual address Dept of Neurorehabilitation, Casa Cura Policlinico, Milan]), Roberto Eleopra (Neurology Unit, S Maria della Misericordia University Hospital, Udine), Nilo Riva (Dept of Neurology IRCCS "San Raffaele" Hospital, Milan), Massimiliano Filosto (Neurologic Clinic, University of Brescia), Fabio Giannini (Dept of Medical and Surgery Sciences and Neurosciences, University of Siena), Enrico Granieri (Neurologic Clinic, University of Ferrara), Vincenzo La Bella (ALS Research Centre, BioNeC, University of Palermo), Giancarlo Logroscino (Dept of Neurology and Psychiatry, University of Bari), Jessica Mandrioli (Dept of Neurosciences, S Agostino-Estense Hospital, Modena), Letizia Mazzini (ALS Centre, Neurologic Clinic, Maggiore della Carità University Hospital, Novara), Maria Rosaria Monsurrò (2nd Neurologic Clinic, 2nd University of Naples, Naples), Vladimiro Pietrini (Dept of Neurosciences, Neurology Unit, University of Parma), Rocco Quatrale (Neurology Unit, dell'Angelo Hospital, Mestre), Romana Rizzi (Neurology Unit, Dept of Neuro-Motor Diseases, IRCCS Arcispedale Santa Maria Nuova, Reggio Emilia), Fabrizio Salvi (IRCCS Neurological Sciences Institute, Bologna), Gabriele Siciliano (Dept of Clinical and Experimental Medicine, Neurology Unit, University of Pisa), Gianni Sorarù (Dept of Neurosciences, University of Padua), Paolo Volanti (Intensive Neurorehabilitation Unit, IRCCS 'Salvatore Maugeri' Foundation, Mistretta).

Contributors GL, IT, AC, GM, GF were involved in the conception, design and interpretation of data. All authors and collaborators of the EPOS Trial Study Group were involved in the collection of data. GL, IT were involved in the drafting of paper. All authors were involved in the approval of final version.

Competing interests None declared.

\section{Patient consent Obtained.}

Ethics approval Ethic Committee at each site.

Provenance and peer review Not commissioned; externally peer reviewed.

Open Access This is an Open Access article distributed in accordance with the Creative Commons Attribution Non Commercial (CC BY-NC 4.0) license, which permits others to distribute, remix, adapt, build upon this work non-commercially, and license their derivative works on different terms, provided the original work is properly cited and the use is non-commercial. See: http://creativecommons.org/ licenses/by-nc/4.0/

\section{REFERENCES}

1 Chio A, Hammond ER, Mora G, et al. Development and evaluation of a clinical staging system for amyotrophic lateral sclerosis. J Neurol Neurosurg Psychiatry 2015;86:38-44.

2 Hardiman 0, van den Berg LH, Kiernan MC. Clinical diagnosis and management of amyotrophic lateral sclerosis. Nat Rev Neurol 2011;7:639-49. 
3 Cedarbaum JM, Stambler N, Malta E, et al. The ALSFRS-R: a revised ALS functional rating scale that incorporates assessments of respiratory function. BDNF ALS Study Group (Phase III). J Neurol Sci 1999;169:13-21.

4 Beghi E, Pupillo E, Bonito V, et al. Randomized double-blind placebo-controlled trial of acetyl-L-carnitine for ALS. Amyotroph Lateral Scler Frontotemporal Degener 2013;14:397-405.

5 Cudkowicz ME, van den Berg LH, Shefner JM, et al. Dexpramipexole versus placebo for patients with amyotrophic lateral sclerosis (EMPOWER): a randomised, double-blind, phase 3 trial. Lancet Neurol 2013;12:1059-67.

6 Lenglet $\mathrm{T}$, Lacomblez $\mathrm{L}$, Abitbol JL, et al. A phase II-III trial of olesoxime in subjects with amyotrophic lateral sclerosis. Eur J Neurol 2014;21:529-36.

7 Sacca F, Quarantelli M, Rinaldi C, et al. A randomized controlled clinical trial of growth hormone in amyotrophic lateral sclerosis: clinical, neuroimaging, and hormonal results. J Neurol 2012;259:132-8.

8 Shefner JM, Watson ML, Meng L, et al. A study to evaluate safety and tolerability of repeated doses of tirasemtiv in patients with amyotrophic lateral sclerosis. Amyotroph Lateral Scler Frontotemporal Degener 2013;14:574-81.

9 Franchignoni F, Mora G, Giordano A, et al. Evidence of multidimensionality in the ALSFRS-R Scale: a critical appraisal on its measurement properties using Rasch analysis. J Neurol Neurosurg Psychiatry 2013;84:1340-5.

10 Voustianiouk A, Seidel G, Panchal J, et al. ALSFRS and appel ALS scores: discordance with disease progression. Muscle Nerve 2008;37:668-72.

11 Wicks P, Massagli MP, Wolf $C$, et al. Measuring function in advanced ALS: validation of ALSFRS-EX extension items. Eur J Neurol 2009;16:353-9.

12 Lauria G, Dalla Bella E, Antonini G, et al. Erythropoietin in amyotrophic lateral sclerosis: a multicentre, randomised, double blind, placebo controlled, phase III study. J Neurol Neurosurg Psychiatry 2015;86:879-86.

13 Cudkowicz ME, Titus S, Kearney M, et al. Safety and efficacy of ceftriaxone for amyotrophic lateral sclerosis: a multi-stage, randomised, double-blind, placebocontrolled trial. Lancet Neurol 2014;13:1083-91.

14 Dupuis L, Dengler R, Heneka MT, et al. A randomized, double blind, placebocontrolled trial of pioglitazone in combination with riluzole in amyotrophic lateral sclerosis. PLOS ONE 2012;7:e37885.

15 Pascuzzi RM, Shefner J, Chappell AS, et al. A phase II trial of talampanel in subjects with amyotrophic lateral sclerosis. Amyotroph Lateral Scler 2010;11:266-71.
16 UKMND-LiCALS Study Group; Morrison KE, Dhariwal S, et al. Lithium in patients with amyotrophic lateral sclerosis (LiCALS): a phase 3 multicentre, randomised, double-blind, placebo-controlled trial. Lancet Neurol 2013:12:339-45.

17 Verstraete $\mathrm{E}$, Veldink $\mathrm{JH}$, Huisman $\mathrm{MH}$, et al. Lithium lacks effect on survival in amyotrophic lateral sclerosis: a phase Ilb randomised sequential trial. J Neurol Neurosurg Psychiatry 2012;83:557-64.

18 Miller RG, Moore DH, Forshew DA, et al. Phase II screening trial of lithium carbonate in amyotrophic lateral sclerosis: examining a more efficient trial design. Neurology 2011;77:973-9.

19 Chio A, Borghero G, Calvo A, et al. Lithium carbonate in amyotrophic lateral sclerosis: lack of efficacy in a dose-finding trial. Neurology 2010;75: 619-25.

20 Aggarwal SP, Zinman L, Simpson E, et al. Safety and efficacy of lithium in combination with riluzole for treatment of amyotrophic lateral sclerosis: a randomised, double-blind, placebo-controlled trial. Lancet Neurol 2010;9:481-8.

21 Meininger V, Drory VE, Leigh PN, et al. Glatiramer acetate has no impact on disease progression in ALS at 40mg/day: a double- blind, randomized, multicentre, placebo-controlled trial. Amyotroph Lateral Scler 2009;10:378-83.

22 Kaufmann P, Thompson JL, Levy G, et al. Phase II trial of CoQ10 for ALS finds insufficient evidence to justify phase III. Ann Neurol 2009;66:235-44.

23 Sorenson EJ, Windbank AJ, Mandrekar JN, et al. Subcutaneous IGF-1 is not beneficial in 2-year ALS trial. Neurology 2008;71:1770-5.

24 Miller R, Bradley W, Cudkowicz M, et al. Phase II/III randomized trial of TCH346 in patients with ALS. Neurology 2007;69:776-84.

25 Gordon PH, Moore DH, Miller RG, et al. Efficacy of minocycline in patients with amyotrophic lateral sclerosis: a phase III randomised trial. Lancet Neurol 2007;6:1045-53.

26 Rosenfeld J, King RM, Jackson CE, et al. Creatine monohydrate in ALS: effects on strength, fatigue, respiratory status and ALSFRS. Amyotroph Lateral Scler 2008;9:266-72.

27 Mitsumoto H, Brooks BR, Silani V. Clinical trials in amyotrophic lateral sclerosis: why so many negative trials and how can trials be improved? Lancet Neurol 2014;13:1127-38.

28 Swash M. Learning from failed trials in ALS. Lancet Neurol 2007;6:1034-5. 